\title{
INFLUENCE OF ADMIXTURES ON PRODUCTION RATE OF ATOMIC NITROGEN
}

\author{
V. Kudrle, A. TÁlskÝ, A. Kudláč, V. Křápek, J. JanČA \\ Department of Physical Electronics, Masaryk University, \\ Kotlářská 2, 61137 Brno, Czech Republic
}

Received 31 March 2000;

final version 30 May 2000

In this paper, we present the dependencies of atomic nitrogen concentration in the afterglow on the amount of oxygen admixture added to the main gas. It is observed that until a certain threshold, the concentration of atomic nitrogen rises rapidly with the amount of admixture. If even more of the admixture is added, the concentration of nitrogen atoms decreases again. We suggest the explanation of this phenomenon by two antagonistic processes acting together: (i) decrease of the surface recombination coefficient by blocking of active sites and (ii) chemical reaction between $\mathrm{N}$ and $\mathrm{O}_{2}$ in the volume.

\section{Introduction}

The processes present at the interface between the discharge plasma and the solid matter are of great importance for physical properties and parameters of the plasma. In the same time, the plasma itself changes the surface, both its structure and its composition. This "surface plasma treatment" is very important [1] for plasma deposition techniques, where thin coatings with selected parameters are deposited on the substrate. Often the quality of such deposited films is determined by the state of the surface of the substrate.

It is well known that the state of the wall surface in the discharge, i.e. its cleanness, structure, amount and type of adsorbed gases, etc., radically influences the heterogeneous loss processes for various species. Particularly important are the changes of surface recombination rate, which in turn directly influence the production rate of atomic species in plasma sources used for oxidation or nitridation. For example, when a small amount of oxygen is added to a nitrogen before its passage through the discharge, one observes [2] important increase in the concentration of atomic nitrogen in the afterglow. It is generally accepted [3] that surface processes play a dominant role in this effect.

\section{Experimental}

A microwave discharge is produced in a quartz discharge tube with inner diameter of $13 \mathrm{~mm}$ passing through the surfatron cavity. Flowing afterglow may be observed in $1 \mathrm{~m}$ long quartz tube with inner diameter of $8 \mathrm{~mm}$. Plasma is sustained by $100 \mathrm{~W}$ power from $2.45 \mathrm{GHz}$ magnetron.

We employed electron paramagnetic resonance (EPR) spectrometer JEOL JES$3 \mathrm{~B}$ to measure the concentration of atomic nitrogen. This method is based on 
resonance absorption of microwave energy by the transitions between Zeeman split levels [4]. In comparison with other techniques it has a big advantage in its noninvasivity and the ability to determine easily the concentrations of wide range of species. The EPR spectra of the isotopes ${ }^{14} N$ and ${ }^{15} N$ are easily distinguishable due to their different nuclear spin.

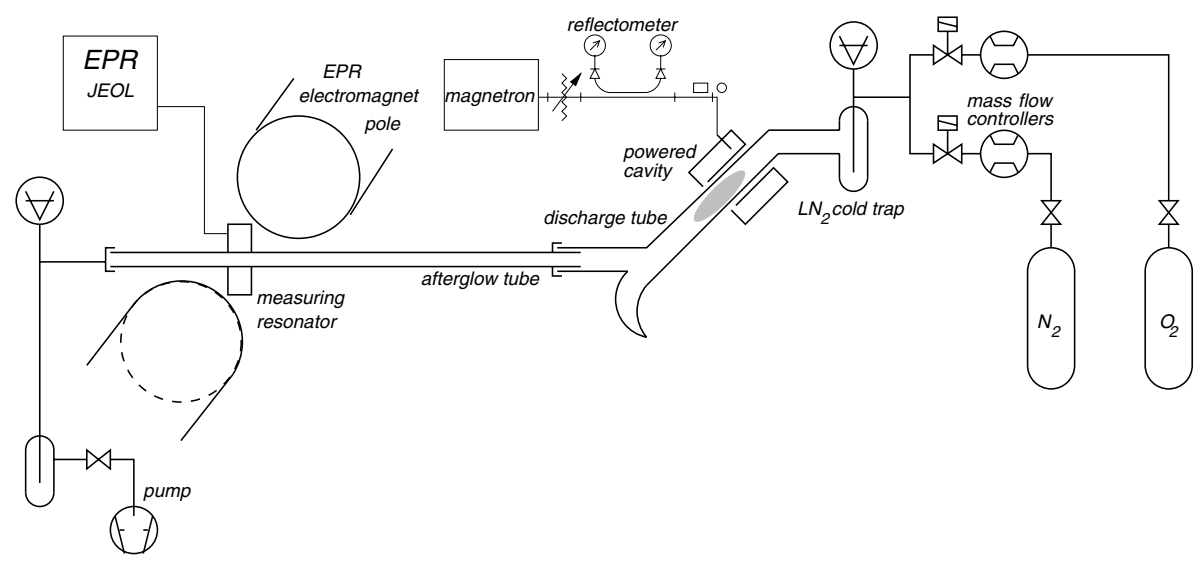

Fig. 1. Schematic drawing of the experimental apparatus.

Our experiments are carried out at nitrogen flow of $50 \mathrm{sccm}$, which corresponds to a pressure of $450 \mathrm{~Pa}$ in the discharge area. The amount of oxygen admixture (up to several percents) is controlled by mass flowcontroller. The concentrations of both atomic nitrogen isotopes ${ }^{15} N$ and ${ }^{14} N$ are determined at afterglow position $27 \mathrm{~cm}$ from the discharge.

\section{Results and discussion}

In the Fig. 2 there is plotted the concentration of atomic nitrogen on the amount of oxygen admixture. One observes that after rapid rise the maximum is reached and for higher abundances of oxygen the atomic nitrogen concentration decreases again. This behaviour can not be explained, when the most common volume reactions [5] are taken into account and thus surface reactions must be introduced.

Let us consider the plasma consisting mainly of the nitrogen gas with small amount of oxygen impurity. The processes taken into account are surface recombination of atomic nitrogen and volume reaction between $\mathrm{N}$ and $\mathrm{O}_{2}$. Therefore the change in concentration of atomic nitrogen is

$$
\frac{\mathrm{d}[N]}{\mathrm{d} t}=C-k_{\mathrm{s}}[N]-k_{\mathrm{v}}[N]\left[O_{2}\right]
$$

where $C$ is the production term, $k_{\mathrm{s}}$ is the rate constant for surface recombination and $k_{\mathrm{v}}$ is responsible for a loss of atomic nitrogen by the reactions with oxygen in the volume. 


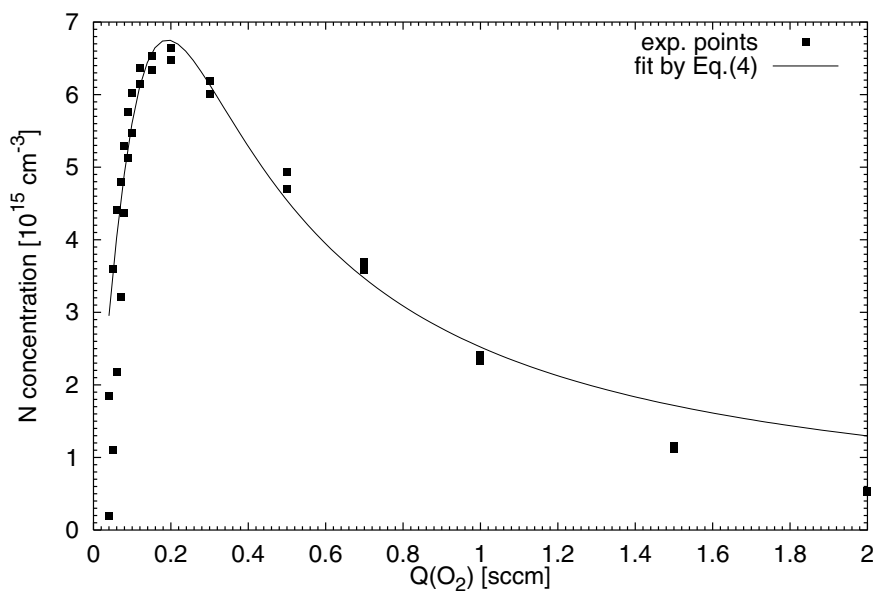

Fig. 2. Dependence of atomic nitrogen concentration on the oxygen admixture.

It is generally supposed [6] that recombination of atoms takes place at so called active sites at the surface. If the active sites become occupied by the molecules of admixture, their effective number decreases and wall recombination coefficient decreases also. From the simple reasoning (Langmuir kinetics) we can conclude that the occupation of active sites by atoms or molecules of admixture should follow

$$
S_{\mathrm{p}}=S_{\mathrm{t}} \frac{\left[\mathrm{O}_{2}\right]}{\left[\mathrm{O}_{2}\right]+b}
$$

where $S_{\mathrm{p}}$ is number of passivated sites, $S_{\mathrm{t}}$ is the total number of active sites and $b$ is certain constant. Now the rate at which the surface recombination takes place may be expressed as

$$
k_{\mathrm{s}}=k_{\mathrm{s}}^{\prime}\left(1-\frac{\left[O_{2}\right]}{\left[O_{2}\right]+b}\right)=\frac{k_{\mathrm{s}}^{\prime} b}{\left[O_{2}\right]+b}
$$

Introducing this into Eq.(1) and searching for steady state $(t \rightarrow \infty)$ solution, the equilibrium concentration of atomic nitrogen is

$$
[N]=\frac{C}{k_{\mathrm{s}}^{\prime} b /\left(\left[O_{2}\right]+b\right)+k_{\mathrm{v}}\left[O_{2}\right]}
$$

By fitting of experimental data (see Fig. 2) by this equation we obtain the values $k_{\mathrm{v}} / C=1.76 \cdot 10^{-16} \mathrm{~cm}^{6}, k_{\mathrm{s}}^{\prime} / C=2.60 \mathrm{~cm}^{3}$ and $b=1.23 \cdot 10^{13} \mathrm{~cm}^{-3}$.

In the Fig. 3 we can observe interesting fact, that the isotope ratio of atomic nitrogen is changing with the amount of admixture. 
$V$. Kudrle et al.: Influence of admixtures on production rate of atomic nitrogen

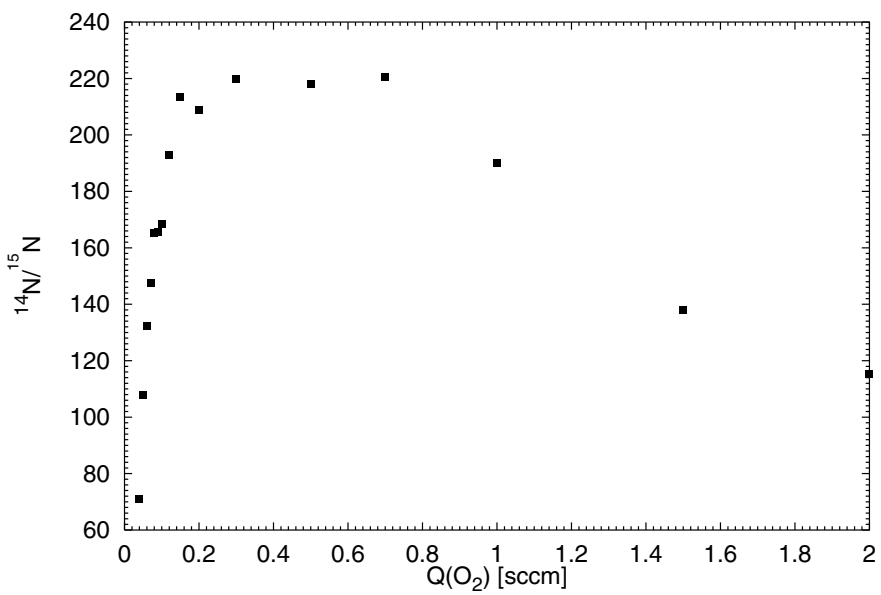

Fig. 3. Influence of oxygen admixture on atomic nitrogen isotope ratio.

\section{Conclusion}

Our experimental results and theoretical analysis suggest that the production of atomic nitrogen is governed by both surface and volume processes. At low concentrations of impurities, the term decreasing the wall recombination coefficient is dominant but at higher admixture flows, the reactions in volume start to decrease again the concentration of nitrogen atoms. For even higher amounts of admixture the more complex phenomena occur which are not well described by our model. Both isotopes of nitrogen $\left({ }^{15} N\right.$ and $\left.{ }^{14} N\right)$ behave similarly but their ratio is changing with the amount of the oxygen impurity.

This work was supported by Grant Agency of the Czech Republic under the contract No.106/96/K245 and by Czech Ministry of Education under the contract No.VS96084.

\section{References}

[1] M.A. Lieberman and A.J. Lichtenberg: Principles of plasma discharges and materials processing. J. Wiley \& Sons, New York, 1994.

[2] V. Zvoníček: in Proceedings of ICPIG'97, Toulouse 1997, vol.4, p.160.

[3] B. Gordiets et al: J. Phys. D: Appl. Phys. 29 (1996) 1021.

[4] A.A. Westenberg: Prog. React. Kinet. 7 (1973) 23.

[5] J. Nahorny: Thesis, Université Paris Sud (1994).

[6] A. Zangwill: Physics at surfaces. Cambridge University Press, 1992, p.206. 